\title{
Image Analysis of Eccentric Photorefraction
}

\author{
J. Dušek, M. Dostálek
}

This article deals with image and data analysis of the recorded video-sequences of strabistic infants. It describes a unique noninvasive measuring system based on two measuring methods (position of I. Purkynje image with relation to the centre of the lens and eccentric photorefraction) for infants. The whole process is divided into three steps. The aim of the first step is to obtain video sequences on our special system (Eye Movement Analyser). Image analysis of the recorded sequences is performed in order to obtain curves of basic eye reactions (accommodation and convergence). The last step is to calibrate of these curves to corresponding units (diopter and degrees of movement).

Keywords: eccentric photorefraction, purkynje images, strabismus, image analysis.

\section{Notation}

PI Purkynje image

ROI Region of interest

COL Centre of lens

$x_{t} \quad$ Co-ordinate of the centre of the lens

$n \quad$ Number of pixels

$j, m \quad$ Pixel position

$d(x) \quad$ Value of the general difference at point $x$

$L \quad$ Half width of the weighting window

$k \quad$ Point of the surroundings and its weight

y Polynomial of interpolation

$a_{0}, a_{1}$ Polynomial coefficients

\section{Introduction}

Accommodation and convergence are synkinetic ocular reflex actions co-ordinated by high brain controllers. Convergence-accommodation synkinesis is a fundamental prerequisite for single binocular vision. Appropriate co-ordination of the two actions is fundamental. There is clinical evidence for the presumption that co-ordination of the two parts of synkinesis is tuned in early infancy. Disturbance of this co-ordination may lead to strabismus. The likehood of successful treatment falls with increasing age of the patient. Therefore, it is important to initiate treatment in infant patients. This requires a careful approach: noninvasive and completely automatic. We have designed a new noninvasive system for measuring of basic eye reactions (accommodation and convergence), especially for infants. The system is based on determining the of horizontal position of the I. Purkynje Image (PI) and eccentric photorefraction.

Purkynje images were discovered in 1823 by J. E. Purkynje [1]. Purkynje images are reflections of the light from optical boundaries of the eye, as shown in Fig. 1.

Eccentric photorefraction is a retinal reflex of incoming light. According to the position of the measuring light source and the estimation algorithm of this light reflection from the eye, two principal subtypes of photorefraction are distinguished:

1. Co-axial methods are based on a light source located on the axis of the camera lens. Four $70^{\circ}$ pi-shaped cylinder lenses attached in front of the camera lens were typical for orthogonal modification of the co-axial method, while the defocus of the camera lens is distinctive for isotropic modification of the method.

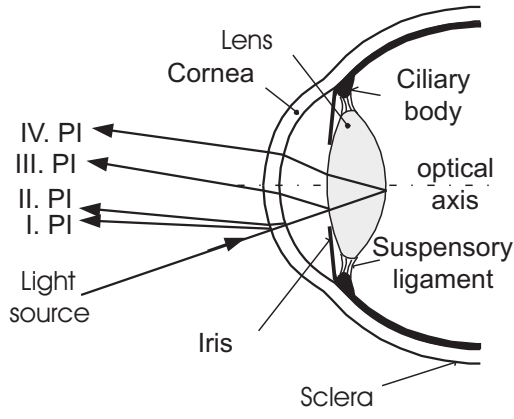

Fig. 1: Formation of Purkynje images

2. Eccentric methods are named after the eccentric position of the measuring light source in front of the camera lens on the shield occluding the part of the lens beneath the light source. The distance between the sharp edge of the shield and the source is called eccentricity and is the crucial parameter of the method. The method was completely described by Bobier and Braddick in 1985 [2]. A principal methodological improvement was brought about by Schaeffel (1987) [3], when the light source design was changed from a point source to an array of point sources. Impractical measurement of the light crescent of reflected measuring light in the pupilla was reduced by measuring the slope of the measurement light intensity in the pupilla. Roorda (1997) showed that if the size of the light source is increased, the intensity profiles become more linear and the slope of the reflex changes linearly with the refractive state (accommodation).

\section{Methodology}

\subsection{Measuring system}

For measurement, we designed a special noninvasive system. The scheme of the system is shown in Fig.2.

This system periodically stimulates the patient's eye system with the two fixation pictures displayed on the fixation monitors. The face of the mother is used as the fixation picture. The ratio between accommodation and convergence is given by the geometric position of these monitors. To elimi- 


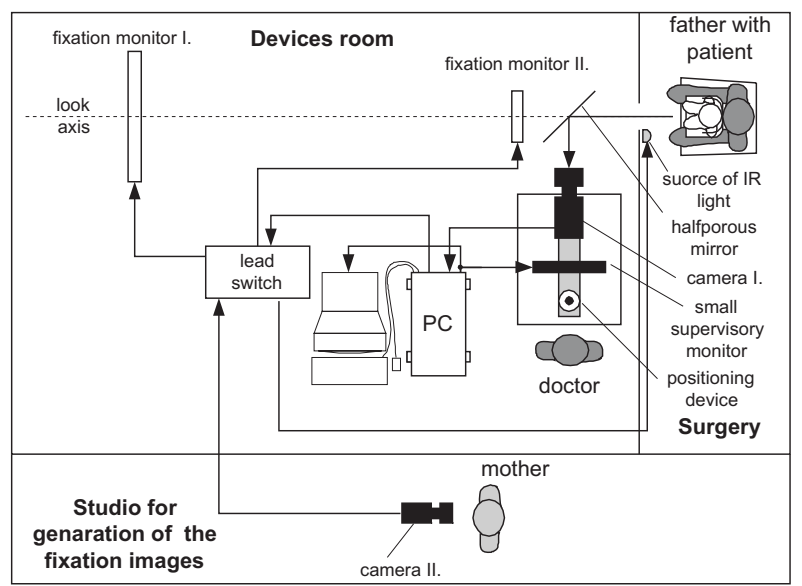

Fig. 2: Scheme of the measuring system

nate the effect of the patient's attention being disturbed, an infrared light point source is used as the measuring light. The doctor finds the position of the eye and starts capturing the picture sequences. The selected capturing camera was DALSA CA D1 with 360 frames per second, which is capable of registering an infrared measuring light.

\subsection{Image analysis}

The image analysis is the same for each picture of the captured sequence.

The first step in our image analysis is to choose a region of interest (ROI) that eliminates an amount of image data (comprising only the lens and necessary surroundings). For subsequent image analysis, the only remaining necessary image data is a rectangular ROI with the lens and part of the iris (see Fig. 3).

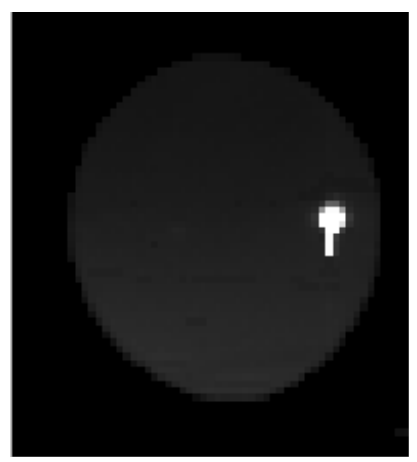

Fig. 3: Extracted ROI with lens and PI

The second step in image analysis is to determine the threshold for partial thresholding [5]. For automatic setting of the threshold we choose another smaller rectangular ROI on the border of the lens and the iris. In this ROI, the program finds the minimum and maximum value, and computes the average value that is set as the threshold. Finally, partial thresholding for the ROI is applied.

The third step is 8-neighborhood identification (for more details see [5]) that controls and labels shapes in ROI and removes any possible undesirable objects or areas other than the lens because of head and eye movements. Then the image analysis is divided into two parts. The fist parts is for convergence, and the second is for accommodation.

For convergence analysis it is necessary to find the horizontal position of the center of the lens, using the following equation

$$
x_{t}=\frac{1}{n} \sum_{j, k} x_{j}(j, m),
$$

where $n$ is the number of pixels, $x_{j}$ is value $j$-position $(j, m)$ pixel of the shape in the picture, $n$ is number of pixels in the object, $x_{t}$ is the co-ordinate of the centre of the lens (COL). The next step determines the horizontal position of PI. First, we do average vertical summation (Fig. 4), which is the vertical summation in the pixel columns divided by the number of nonzero pixels in the same column. Then general difference with a weighting window that eliminates local extremes is applied twice, where $x$ is the point where the general difference is

$$
d(x)=\frac{\sum_{k=-L}^{L} k f(x+k)}{\sum_{k=-L}^{L} k^{2}},
$$

computed, $d(x)$ is the value of the general difference at point $x, L$ is half of the width of the weighting window, $k$ is the point of the surroundings and its weight. Then, we find the minimum zero crossing point in first difference and in the second difference we find the curve crossing point that of this curve that represents maximum of the original curve - the global extreme. In order to achieve precision, we interpolate the surroundings of this extreme. The result is the horizontal position of PI.

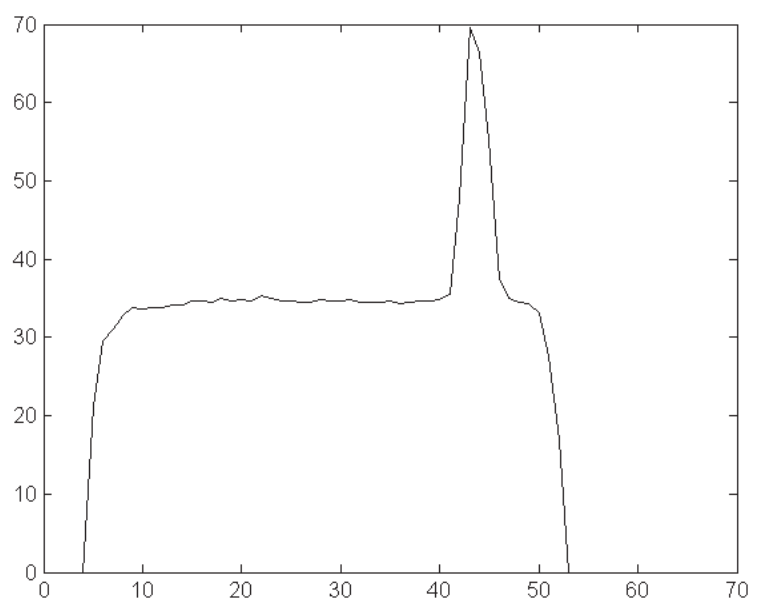

Fig. 4: Average vertical summation

For convergence analysis we subtract PI and $x_{j}$, which represents the distance between PI and COL and shows us the time demanding process of convergence.

The first step in accommodation analysis is to remove PI from the thresholded ROI by a new partial thresholding. The new threshold is set at $70 \%$ of the dynamic range, which eliminates higher values of brightness that represent I. PI. 
The average horizontal summation (the same as in convergence analysis, but in the horizontal direction) is presented in Fig. 5.

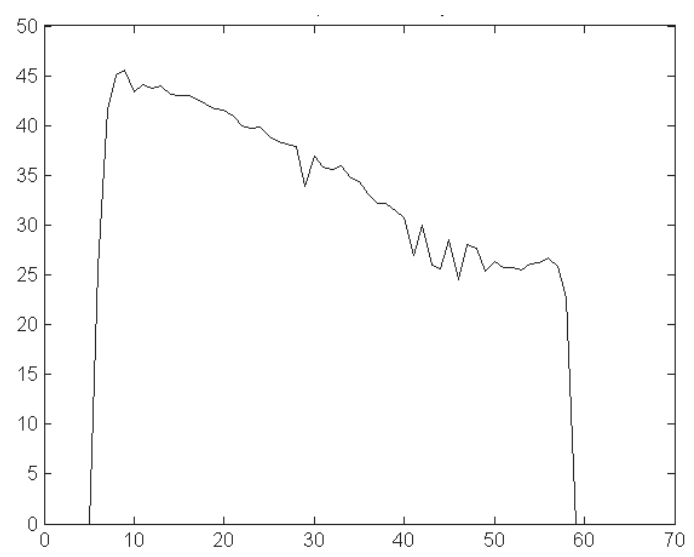

Fig. 5: Average horizontal summation

This is the horizontal summation in pixel rows divided by the number of nonzero pixels in the same row. By fitting the middle part of this curve we get the following polynomial:

$$
y=a_{0}+a_{1} x .
$$

For accommodation analysis we use coefficient $a_{1}$, which that represents the slope of the curve.

\subsection{Calibrating the curves}

The last step involves calibrating of both curves to the corresponding units (diopter and degrees of movement). This calibration depends on the geometrical position of both monitors and patient.

For calibration of accommodation we use distances of the fixation monitors that represent relative defocus, and starting dioptric power that is computed as the average of the first 25 values of $a_{1}$ (before accommodation starts - fixation on the first monitor).

Calibration of the convergence is angle transformation between the view axis of the eye and the camera, and it is done separately for each eye. The range of convergence is given by the position of the fixation monitors. The start angle is computed as the average of the first 25 values of the relative dis- tance between PI and COL (before convergence starts - fixation on the first monitor).

\section{Results}

A unique system for automatic measurement of accommodation and convergence has been designed and implemented, as shown in Fig. 2. Test have shown that the system is capable of detecting I. PI and eccentric retinal refraction. Fig. 3 shows the most important part of the recorded picture (ROI) with high system resolution and sensitivity. The results of automatic data analysis and calibration are presented in Fig. 6 in a graph of the time demanding process of accommodation and convergence.

\section{Conclusions}

We have designed a universal system for measuring of synkinetic reaction (accommodation and convergence) based on I. PI position and eccentric photorefraction. This system has many advantages, e.g., it is noninvasive, automatic, cheap and easy to operate. Its precision is good enough for clinical practice. Using this system we are able to obtain a time curve of synkinetic reaction. These curves enable us to recognize and diagnose a range of eye defects and squints.

\section{Acknowledgment}

This research work has been supported by research program No. MSM 210000012 “Transdisciplinary Biomedical Engineering Research" of the Czech Technical University in Prague (sponsored by the Ministry of Education Youth and Sports of the Czech Republic) and partly supported by grant-founded project GAČR No. 102/00/1494.

\section{References}

[1] Purkyně J. E.: Commentario de examine physiologico organi visus, Breslau, 1823.

[2] Bobier W. R. \& Braddick: "Eccentric photorefraction: Optical analysis and empirical measures". Am. J. Optom. Physiol. Opt., Vol. 62, 1985, p. 614-620.

[3] Schaeffel F., Farkas L., Howland H. C.: "Infrared photoretinoscope”. Appl. Opt., Vol. 26, 1987, p.1505-1509.

\section{Patient's identification mark}

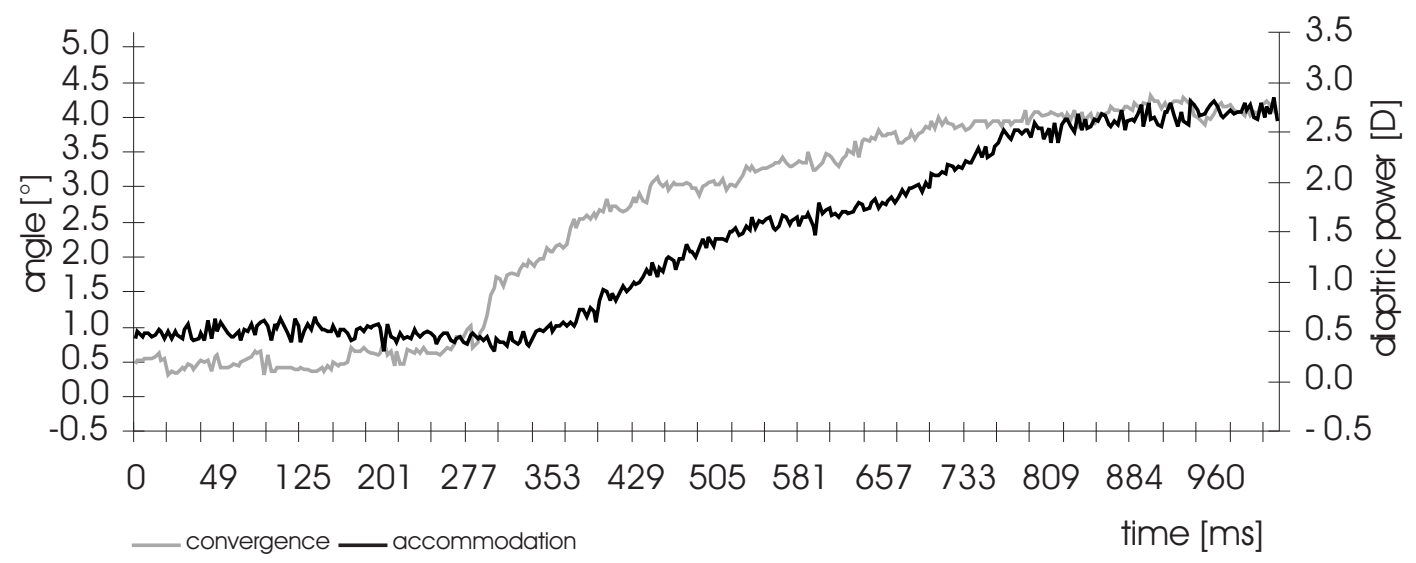

Fig. 6: Results of image analysis 
[4] Roorda A., Campbell M. C. W., Bobier W. R.: "Slope-based eccentric photorefraction: theoretical analysis of different light source configurations and effects of ocular aberrations". J. Opt. Soc. Am. A., Vol. 14, 1997, p.2547-2556.

[5] Šonka M., Hlaváč V., Boyle R.: Image Processing, Analysis and Machine Vision. PWS, Boston, USA, second edition, 1998, p.695.

Ing. Jaroslav Dušek

phone: +420224352113

fax: +420233339801

e-mail:xdusekj@feld.cvut.cz

Department of Radioelectronics

Czech Technical University

Faculty of Electrical Engineering

Technická 2

16800 Prague 6, Czech Republic

MUDr. Miroslav Dostálek

phone: +420604148517

e-mail:dostalek@lit.cz

Department of Ophthalomology

Litomyšl Hospital

Purkyňova 919

7001 Litomyšl, Czech Republic 